\title{
Exhaustive and Efficient Identification of Rationales Using GQM+Strategies with Stakeholder Relationship Analysis*
}

\author{
Takanobu KOBORI $^{\dagger a)}$, Nonmember , Hironori WASHIZAKI ${ }^{\dagger b)}$, Yoshiaki FUKAZAWA ${ }^{\dagger c)}$, Members, $^{\text {(c) }}$ \\ Daisuke HIRABAYASHI $^{\dagger \dagger \mathrm{d})}$, Katsutoshi SHINTANI ${ }^{\dagger \dagger \dagger \mathrm{e}}$, Yasuko OKAZAKI ${ }^{\dagger \dagger \dagger \mathrm{f})}$, \\ and Yasuhiro KIKUSHIMA ${ }^{\dagger \mathrm{g})}$, Nonmembers
}

\begin{abstract}
SUMMARY To achieve overall business goals, GQM+Strategies is one approach that aligns business goals at each level of an organization to strategies and assesses the achievement of goals. Strategies are based on rationales (contexts and assumptions). Because extracting all rationales is an important process in the GQM+Strategies approach, we propose the Context-Assumption-Matrix (CAM), which refines the GQM+Strategies model by extracting rationales based on analyzing the relationships between stakeholders, and the process of using GQM+Strategies with CAM effectively. To demonstrate the effectiveness of the CAM and the defined process, we conducted three experiments involving students majoring in information sciences at two different Japanese universities. Moreover, we applied the GQM+Strategies approach with CAM to the Recruit Sumai Company in Japan. The results reveal that compared to GQM+Strategies alone, GQM+Strategies with CAM can extract rationales of the same quality more efficiently and exhaustively.
\end{abstract}

key words: stakeholder, actor, GQM+Strategies, context, assumption

\section{Introduction}

Because software is responsible for a lot business in corporate activities [3] and the complexity of software and IT systems has been increasing, linking business and system requirements is becoming more difficult. Often it is unclear if IT/software related strategies and organizational business goals are aligned. According to V. Mandić et al. [4],

\section{Manuscript received January 13, 2016.}

Manuscript revised June 9, 2016.

Manuscript publicized July 6, 2016. Japan.

${ }^{\dagger}$ The authors are with Waseda University, Tokyo, 169-8555

${ }^{\dagger}$ The author is with the T\&D INFORMATION SYSTEM Ltd., Saitama-shi, 330-0075 Japan.

t†T The author is with the Software Engineering Center Information-technology Promotion Agency, Tokyo, 113-6591 Japan.

掠 The author is with the IBM, Tokyo, 103-8510 Japan.

*This paper is an extended version of a paper presented at the 1st Asia Pacific Requirements Engineering Symposium [1] and the 15th International Conference of Product Focused Software Development and Process Improvement [2]. We have added some descriptions that propose the process of using GQM+Strategies with CAM. Moreover, we have added some discussions to the section of evaluation.

a)E-mail: uranus-tk@ruri.waseda.jp

b)E-mail: washizaki@waseda.jp

c) E-mail: fukazawa@waseda.jp

d) E-mail: dieten10@yahoo.co.jp

e) E-mail: katsu.shintani@k3.dion.ne.jp

f)E-mail: yokazaki@jp.ibm.com

g) E-mail: y-kiku@ark.ocn.ne.jp

DOI: 10.1587/transinf.2015KBP0009 the success of measurement initiatives in software companies depends on the quality of the links between the metrics programs and organizational business goals. One approach to resolve this issue is GQM+Strategies $\AA$ [5], [6], which aligns and assesses the business goals at each level to the overall strategies and goals of the organization**. Many companies worldwide (e.g., the Japan Aerospace Exploration Agency [7], the global oil and gas industry [8], and non-software development domains such as the military training domain [9]) have applied GQM+Strategies for measurement-based IT-business alignment.

GQM+Strategies has been used to establish management strategies and plans, determine the value of a contribution, ensure the integrity of a goal between a purchaser and a contractor, and evaluate management based on quantitative data. It extracts strategies from goals based on rationales (contexts and assumptions). However, the lack of rationales tends to be misleading and may result in deriving incorrect strategies. Although rationales must be identified exhaustively to extract valid strategies, it is often unclear whether the identified rationales cover all existing ones. Moreover, rationales tend to be extracted from the analyst's viewpoint, although the viewpoints of all stakeholders are also important to consider. Many rationales should be extracted to ensure all elements are used to define goals and strategies. If there are only a few rationales, strategies must be extracted from limited number of rationales. Similarly, strategies must be extracted from a few rationales if the rationales are numerous but low quality. These situations may lead to a misunderstanding in the meaning of the rationale, which may result in extracting the wrong strategy. Therefore, all the rationales should be high quality to maintain consistency. Herein the quality of rationales means that a clear and detailed description helps derive the goals and strategies.

This paper proposes the Context-Assumption-Matrix (CAM) to refine business goals and strategies iteratively [1], [2]. Moreover, we define the process to apply CAM to GQM+Strategies. CAM should extract many rationales from multiple viewpoints. For example, the balance scorecard [10] addresses the importance of considering the organizational business goals from four perspectives multilaterally. In this paper to answer RQ1 and RQ2, we confirm this

${ }^{* *} \mathrm{GQM}+$ Strategies ${ }^{\circledR}$ is registered trademark No. 302008021763 at the German Patent and Trade Mark Office; international registration number IR992843 
assertion in three experiments. Considering rationales multilaterally using CAM might affect the quality of rationales on the whole. There is concern that CAM might reduce the quality of rationales when applied in a limited time. In this paper, we address this issue in RQ3 using three experiments.

Herein four research questions are examined.

- RQ1: Can using GQM+Strategies with CAM extract more rationales efficiently than using only GQM+Strategies?

- RQ2: Can using GQM+Strategies with CAM extract rationales from more viewpoints than using only $\mathrm{GQM}+$ Strategies?

- RQ3: Does using GQM+Strategies with CAM extract higher quality rationales than using only GQM+Strategies?

- RQ4: Can using GQM+Strategies with CAM support to plan the actual goals and strategies of an organization?

The contributions of this paper are two-fold. First, the proposed method may provide an efficient and exhaustive method to extract contexts and assumptions of the same quality as GQM+Strategies alone. Second, in three experiments and an application to the actual company, we confirm the defined process of applying CAM to GQM+Strategies is useful. The rest of the paper is structured as follows. Section 2 overviews the GQM+Strategies approach and provides a motivating example of our approach. Section 3 explains our approach. Sections 4 and 5 introduce case studies. Section 6 explains the limitation of our experiments. Section 7 discusses related works. Finally, Sect. 8 concludes the paper and suggests future work.

\section{Background}

\subsection{GQM+Strategies}

GQM+Strategies was initially developed by the Fraunhofer Center for Empirical Software Engineering (CESE) [11] and Fraunhofer Institute for Experimental Software Engineering (IESE) [12]. This approach extends the goal/ question/ metric paradigm to measure the success or failure of goals and strategies, while adding enterprise-wide support to determine actions on the basis of the measurement results [6], [13]. Collecting data to measure the success or failure of goals and strategies is especially difficult when developers do not know which data are necessary [14]. GQM assists developers by creating software-related goals, generating questions to refine goals, and specifying measures that must be considered to answer the generated questions [8]. Although the GQM approach can measure whether a business goal is achieved in an organization, it lacks a mechanism to link higher- and lower-level business goals. Consequently, it cannot support and integrate goals at different levels of an organization.

On the other hand, GQM+Strategies creates maps between goal-related data at different levels, allowing insights

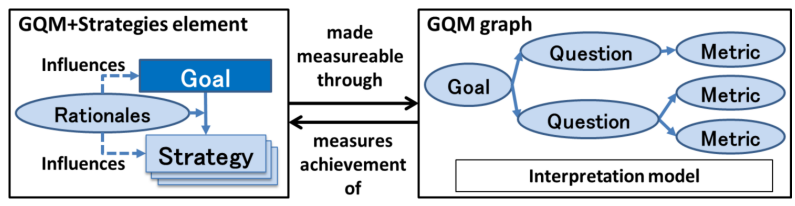

Fig. 1 GQM+Strategies components

gained relative to a goal at a lower level to satisfy goals at higher levels [15]. The major feature of GQM+Strategies is that business goal strategies are determined based on rationales as "contexts" and "assumptions". Contexts are environmental characteristics, while assumptions are aspects of uncertain environments, including estimated ones. After considering many strategies for a goal, the best one is then selected based on the rationales. Because all of the selected strategies are detailed into lower level goals, it is possible to determine strategies that reflect the actual business environment. Figure 1 overviews the concept of GQM+Strategies. The GQM+Strategies Grid visually confirms the link between a goal and a strategy, allowing the entire organization to communicate easily and work toward a common goal. Furthermore, through the GQM paradigm, whether goals at each level are achieved can be evaluated. Our approach uses the following terminology (based on Basili et al. [8], [16]):

- Organizational goal: Objective to accomplish in a given time frame that encompasses part or all of the organization.

- Strategy: Possible approach to achieve a goal within the organization's environment. The number of strategies depends on the internal structure of an organization.

- Rationale: Relevant context or assumption used to select goals and strategies.

- Context: External or internal organizational environment.

- Assumption: Estimated unknown.

\subsection{Motivating Examples}

To successfully adapt GQM+Strategies, it is important to capture rationales. High-quality GQM+Strategies Grids can guide an organization and help achieve business goals and strategies. However, this ability depends on the methods to "capture" the relevant context (internal and external environments) [17].

As an example, we applied GQM+Strategies to the sales department of a stationary company, which sells stationary to corporations. The company receives orders from corporate customers and then ships based on the order form. The corporate structure of the stationary company has three level (top management level, department level, and group level). The purpose of using GQM+Strategies is to improve the order acceptance process of the sales department and the shipping business.

Figure 2 shows the group level business goal, strategy, 


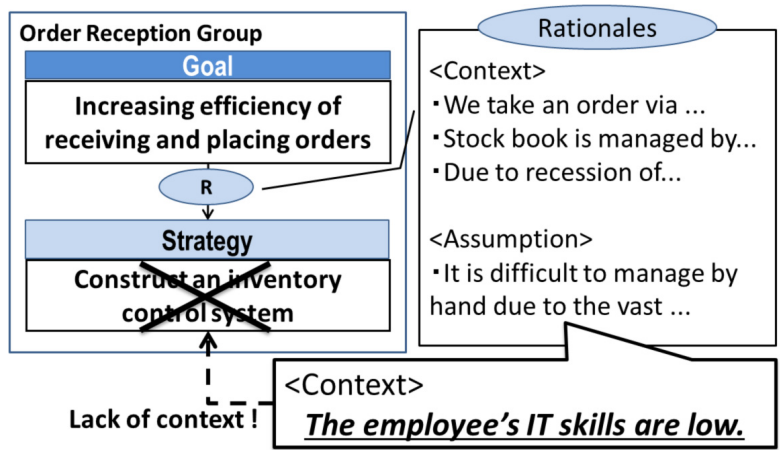

Fig. 2 Goal, strategy, and rationales (excerpt)

and rationales. The strategy, which constructs an inventory control system, is extracted from the goal to increase the efficiency of the order reception business. Although the GQM+Strategies process derives business goals, strategies, and rationales, it is unclear whether the contexts and assumptions cover all existing goals and strategies. For example, there may be a context that limits the budget, making the strategy determined in Fig. 2 impossible to execute. The lack of contexts and assumptions tends to be misleading and may result in deriving incorrect strategies. Therefore, a mechanism to extract contexts and assumptions efficiently and exhaustively is necessary.

\section{Our Approach}

In Sect. 3.1, we propose the CAM, which is a method to extract contexts and assumptions efficiently and exhaustively by analyzing the relationships between stakeholders. In Sect.3.2, we describe how to use GQM+Strategies with CAM.

\subsection{Context-Assumption-Matrix}

The CAM organizes common contexts and assumptions between stakeholders into a two-dimensional table. Our approach defines stakeholders as people, systems, or processes, which enables the CAM to respond to the actual shape of a corporation. Figure 3 provides an example when the CAM is applied to the GQM+Strategies Grid of the stationary company in the motivating example. Each row element denotes a stakeholder who views the context or assumption. Each column element represents a stakeholder who is the subject of the context or assumption. For example, C3 (Context 3) in Fig. 3 is the "inventory control group sometimes mistakes the number of the stock". This means that the "order reception group" (row) views that the "inventory control group" (column) takes an order. The inventory control group's row in Fig. 3 shows that this row lacks contexts or assumptions related to the inventory control group. Thus, the contexts and assumptions from the viewpoint of the inventory control group may be omitted. In fact, there is a context, "the employee's IT skills are low". Thus, the CAM can extract contexts and assumptions.

\begin{tabular}{|c|c|c|c|c|c|}
\hline Viewpoint & $\begin{array}{l}\text { Order reception } \\
\text { Grp. }\end{array}$ & $\begin{array}{l}\text { Shipment } \\
\text { Grp. }\end{array}$ & $\begin{array}{c}\text { Inventory Control } \\
\text { Grp. }\end{array}$ & $\cdots$ & TBD \\
\hline $\begin{array}{l}\text { Order } \\
\text { reception } \\
\text { Grp. }\end{array}$ & 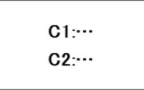 & & $\begin{array}{l}\text { C3:Inventory control group } \\
\text { sometimes mistake the } \\
\text { number of the stocks. }\end{array}$ & & $\begin{array}{l}\text { C4: No one integrates } \\
\text { complaints from } \\
\text { customers in } \\
\text { customer service }\end{array}$ \\
\hline $\begin{array}{l}\text { Shipment } \\
\text { Grp. }\end{array}$ & & $\begin{array}{l}\text { C5: } \cdots \\
\text { A1: } \cdots\end{array}$ & A2: $\cdots$ & & \\
\hline \multirow{2}{*}{\multicolumn{6}{|c|}{\begin{tabular}{|c|} 
Inventory \\
Control Grp. \\
$\ldots$ \\
\end{tabular}}} \\
\hline & & & & & \\
\hline TBD & & & & & \\
\hline
\end{tabular}

Fig. 3 Example of applying the CAM to a stationary company

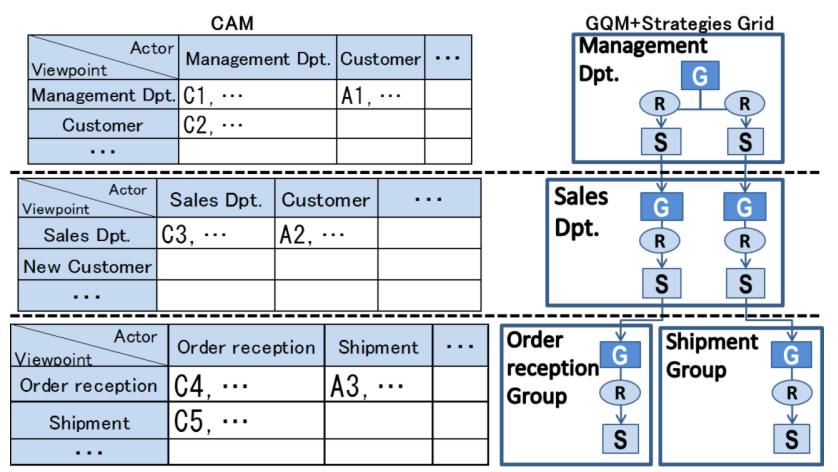

Fig. 4 Structure of the CAM and the GQM+Strategies Grid for the stationary company

Moreover, the CAM has a column labeled TBD, which stands for To Be Determined. In the CAM, TBD represents a stakeholder who is undecided or does not currently exist. For example, C4 (Context 4) in Fig. 3 is "no one integrates complaints from customers in customer service", which indicates that this role is not currently assigned. The rationales in TBD may create new strategies. For example, they introduce Customer Relationship Management.

Figure 4 shows the structure of the CAM and the GQM+Strategies Grid for the aforementioned stationary company. Similar to the GQM+Strategies Grid, the CAM has a hierarchy corresponding to the corporate structure. In this case, the CAM has three levels because the example stationary company has three levels. The stakeholders of the CAM have the same levels as the corporate structure. Initially, the stakeholders of the CAM are determined based on the corporate structure (i.e., the Management Department is level 1, the Sales Department is level 2, and the Order Reception Group and the Shipment Group are level 3), but new stakeholders (e.g., for operations and maintenance) can be added as necessary. The lower and upper levels are assumed to have the same rationales. In this case, rationales at a higher level are defined abstractly for the management department, while the ones at the lower level are defined concretely for the level groups. Hence, the CAM allows the contexts and assumptions to be visually reviewed.

\subsection{Process of Using GQM+Strategies with CAM}

The CAM has difficulty extracting rationales from the viewpoints of undefined stakeholders. Our preliminary experiments revealed that if a CAM cell contains a rationale, it 
assumes that all rationales have been completely extracted. However, this deficiency can be resolved by reapplying the CAM after considering the viewpoints of undefined stakeholders freely in a brainstorming meeting. Therefore, we define the process of using GQM+Strategies with CAM (Fig. 5). GQM+Strategies is based on Basili et al. [8], [16]. The CAM has two uses; one is to extract missing rationales in the definition process (Step 2, 3, 4), and the other is to check rationales in the revision process (Step 9).

Our approach uses the following steps:

1. Collect the rationales in group brainstorming meetings. Derive as many rationales as possible, welcoming unique and innovative ones.

2. Extract the initial stakeholders of the CAM from the departments and groups based on the organizational structure. For example, we extracted the management department, sales department, order reception group, shipment group, and customer in Fig. 4.

3. Apply the collected rationales to the CAM by emphasizing who views the rationale and who is subject of the rationale. According to these stakeholders, map the rationales in the CAM.

4. Use the CAM to extract missing rationales. If the CAM is a sparse matrix, it is possible rationales are omitted.

5. Define the organizational goals and execute strategy decisions based on the collected rationales.

6. Specify plans to implement the GQM+Strategies in the organization. Develop strategy plans and measurement plans.

7. Execute strategies in step 7, and collect measurement data.

8. Analyze the measurement data to determine the success of the goals and strategies defined the grid.

9. Review the rationales from the viewpoint of all stake-

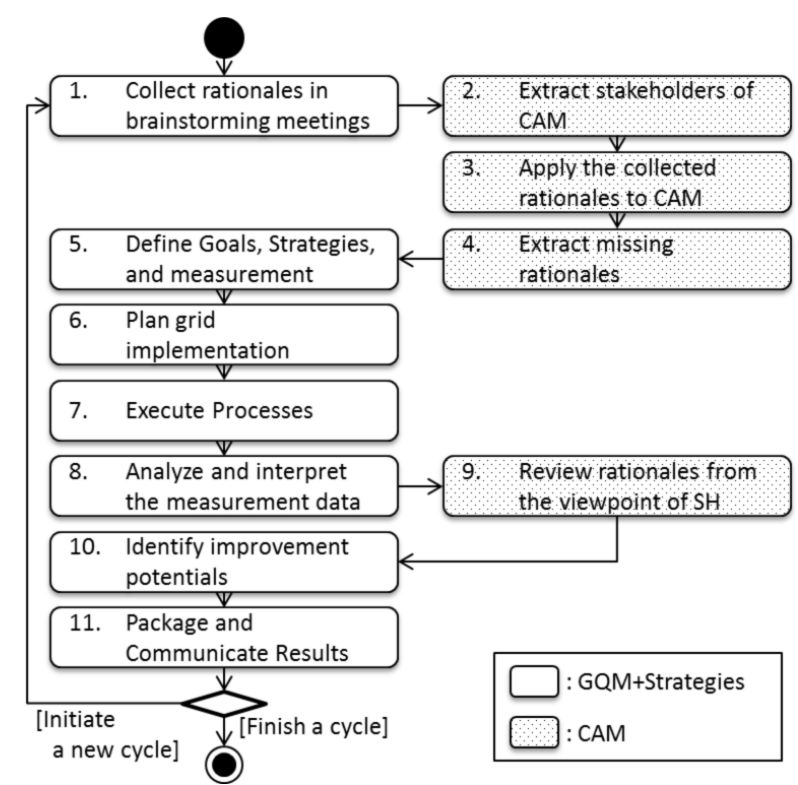

Fig.5 Process of using GQM+Strategies with CAM holders in the CAM. In particular, verify that the rationales in the row of stakeholder related to the goals are achieved in the CAM.

10. Identify potential improvements with respect to the grid and the GQM+Strategies implementation process.

11. Evaluate the results of the above steps. Modify the GQM+Strategies grid in order to close the gaps identified in previous steps.

12. If necessary, initiate a new cycle.

\section{Case Study 1: Application of the CAM in University Courses}

\subsection{Evaluation Design}

To answer RQ1, RQ2 and RQ3, we conducted three control experiments involving students majoring in information sciences at Shimane University and Waseda University (Table 1). In each experiment, the participants were divided into two groups. Both groups listened to a lecture about GQM+Strategies and the CAM. We provided students with a company profile, which explained the management plan, business environment, workflows, and the circumstances surrounding the company. A cosmetic company profile was used to set business goals in a GQM+Strategies Grid. Students were instructed to derive contexts, assumptions, and strategies for the goals. Students extracted rationales based on the profile. They also determined additional rationales freely. Then they derived goals and strategies based on these rationales. Group A experimented with GQM+Strategies only, but Group B experimented with GQM+Strategies and CAM. We conducted three experiments where the deliverable was the GQM+Strategies grid containing goals, strategies, and rationales. Group A (GQM+Strategies team) wrote rationales on paper, whereas Group B (the GQM+Strategies and CAM team) wrote rationales on CAM and then on paper.

At Shimane University, we conducted an experiment involving 43 students majoring in information sciences on the last day of a four-day software engineering class. We divided students into seven teams of five or six people (In the evaluation, one team was excluded because they were late for the lecture). Three teams were in Group A and three were in Group B. The experiment took three hours.

At Waseda University in 2014, we conducted an experiment involving 28 students majoring in information sciences. The experiment was conducted during a software engineering class. We divided students into five teams of five or six people. Group A had three teams and Group B had

Table 1 Experimental settings

\begin{tabular}{crrr}
\hline & Shimane & Waseda 2014 & Waseda 2015 \\
\hline People (number) & 43 & 28 & 32 \\
Time (hours) & 3 & 1 & 1.5 \\
Group A (teams) & 3 & 3 & 3 \\
Group B (teams) & 3 & 2 & 3 \\
\hline
\end{tabular}



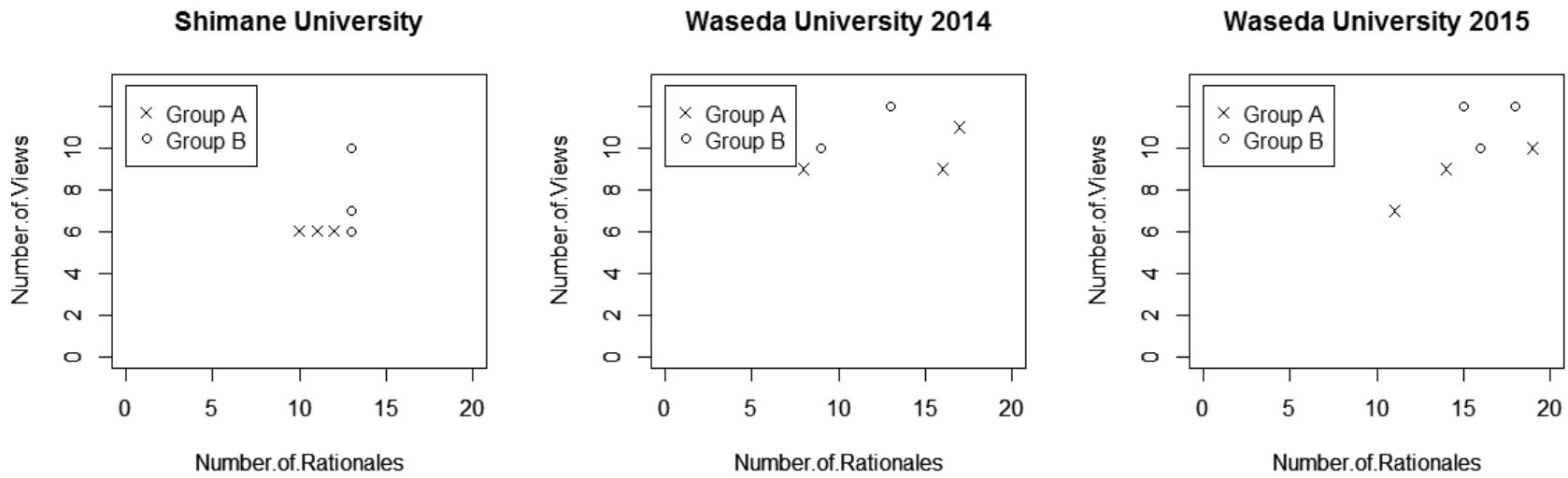

Fig. 6 Results of the three experiments

Table 2 The results of Mann-Whitney's U test

\begin{tabular}{ccccc}
\hline & \multicolumn{2}{c}{ Number of rationales } & \multicolumn{2}{c}{ Number of views } \\
\cline { 2 - 5 } & $\mathrm{W}$ & $\mathrm{p}$-value & $\mathrm{W}$ & $\mathrm{p}$-value \\
\hline Shimane & 0 & 0.064 & 1.5 & 0.20 \\
Waseda 2014 & 4.0 & $\mathbf{0 . 0 8}$ & 1.0 & 0.37 \\
Waseda 2015 & 3.0 & $\mathbf{0 . 7 0}$ & 0.5 & 0.12 \\
\hline
\end{tabular}

Table 3 Evaluation of the assumption's quality (number of good assumptions/total number of assumptions)

\begin{tabular}{cccc}
\hline & Shimane & Waseda 2014 & Waseda 2015 \\
\hline Group A & 0.76 & 0.77 & 0.52 \\
(Good/Total) & $(34 / 45)$ & $(46 / 60)$ & $(11 / 21)$ \\
\hline Group B & 0.80 & 0.67 & 0.83 \\
(Good/Total) & $(36 / 40)$ & $(18 / 27)$ & $(30 / 36)$ \\
\hline
\end{tabular}

two. The experiment took one hour.

At Waseda University in 2015, we conducted an experiment involving 32 students majoring in information sciences. The experiment was conducted during a software engineering class. We divided students into six teams of five or six people. Three teams were in Group A and three teams were in Group B. The experiment took one and a half hours.

\subsection{Experimental Results}

To compare the case using only GQM+Strategies to that using GQM+Strategies with CAM, we mapped the rationales, which Group A extracted using only GQM+Strategies, to the CAM after the experiments. The number of rationales is the sum of the number of context and assumptions extracted by the students, while the number of views is the sum of the number of "viewpoints" and "who" in the CAM. Figure 6 plots the results of each experiment. The X-axis represents the number of rationales, while the Y-axis represents the number of views. The team on the top right of the figure is able to verify and extract rationales from many viewpoints. An "o" represents the teams using GQM+Strategies with CAM, while an " $x$ " denotes the teams using only $\mathrm{GQM}+$ Strategies.

Table 3 evaluates the assumption's quality (number of good assumptions/total number of assumptions). In these experiments, students simply extracted the contexts from the cosmetics company's profile as they determined the assumptions based on the cosmetics company's profile. Therefore, we evaluated only the assumptions that the students extracted. In order to evaluate the quality, we asked three GQM+Strategies experts to evaluate the students' findings based on two grades: good or bad. Grades depended on whether the rationale is appropriate to the business goals and strategies.

\subsection{Discussion}

\section{RQ1: Can using GQM+Strategies with CAM ex- tract more rationales efficiently than using only GQM+Strategies?}

At Waseda University in 2014, Group B, which used both GQM+Strategies and CAM, extracted an average of 2.6 less rationales. This result more likely reflects the time constraint of the experiment, which prevented Group B from efficiently using both GQM+Strategies and CAM. Thus, to demonstrate the effectiveness of the CAM, Group B in the other two experiments used only the CAM. Compared to Group A, Group B at Shimane University (Waseda University in 2015) extracted an average of 2.0 (1.7) more rationales (Fig. 6). The numbers $(2.6,2.0$, and 1.7) mean the difference between the average of teams in Group A and the average of teams in Group B. The averages were calculated for each group. For example, at Shimane University in Fig. 6, Group A found 10, 11, and 12 rationales. Thus, the average number of rationales for Group A was 11. On the other hand, Group B found 13 rationales in each experiment, giving an average of 13 . Therefore, the difference between the two groups is $2.0(=13-11)$.

Because each experiment had different conditions (exercise time, proficiency level), we performed statistical analysis on each experiment separately. Due to the small amount of data, whether the experimental results follow a normal distribution cannot be confirmed. Hence, we performed Mann-Whitney's U test (Wilcoxon rank sum test) since it does not require the assumption of normal distributions. Table 2 shows the results of Mann-Whitney's U test. This table 

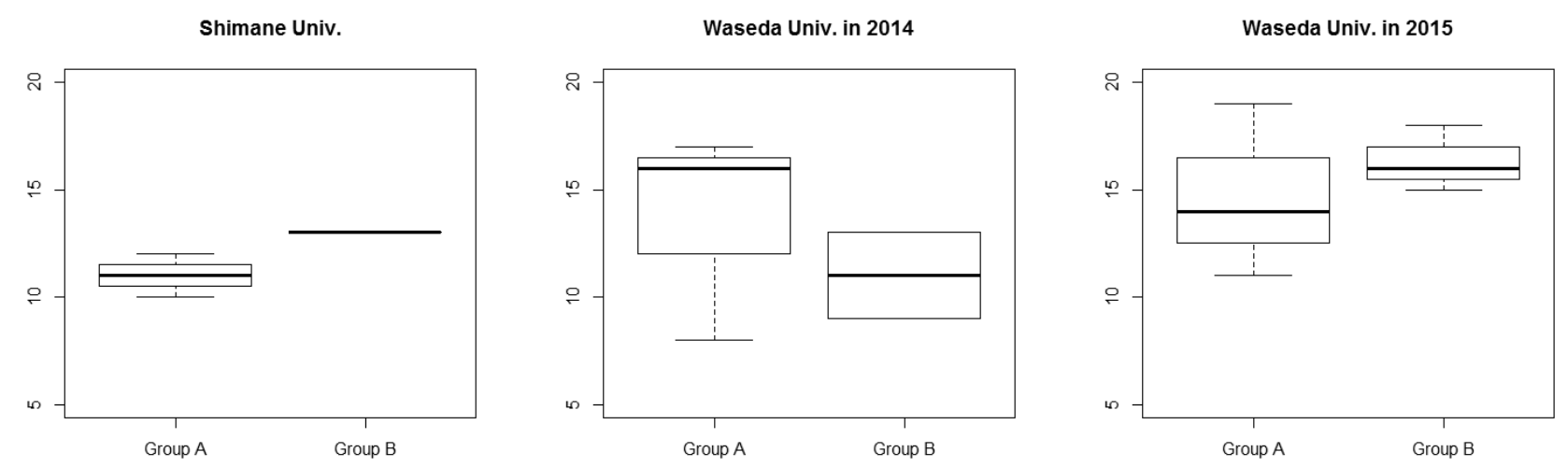

Fig. 7 Results of boxplot on the number of rationales
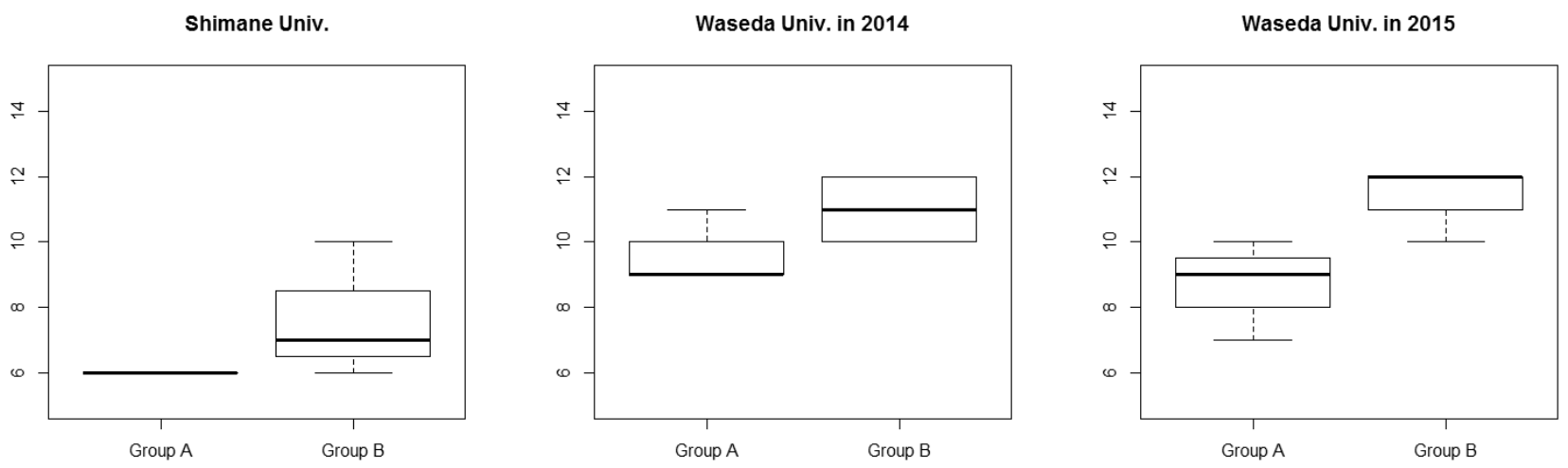

Fig. 8 Results of boxplot on the views of rationales

shows the probability of obtaining the observed or more extreme results when the null hypothesis is true (i.e. distributions are equal) as p-value, and the value of the test statistic as 'W'. Figure 7 shows boxplots of the results on the number of rationales. This figure compare the results between Group A's data and Group B's data. Although the differences are not statistically significant at the $p=0.05$ level in Table 2, there is one case (i.e. Shimane University) showing statistically significant difference in the number of rationales at the $\mathrm{p}=0.1$ level in Table 2. Moreover, regarding the average, the GQM+Strategies and CAM teams (i.e. Group B) have higher averages than the GQM+Strategies teams (i.e. Group A) in Fig. 7. According to these results, it seems that GQM+Strategies with CAM could extract rationales more efficiently than GQM+Strategies alone.

This is because Group A extracted new rationales ad hoc, while Group B extracted them based on "viewpoint" and "actor" in the CAM. The results show that new rationales can be effectively extracted using the CAM when sufficient time is allotted.

\section{RQ2: Can using GQM+Strategies with CAM extract rationales from more viewpoints than using only GQM+Strategies?}

Group B resulted in 1.6 more views at Shimane University, 1.4 more views at Waseda University in 2014, and 2.7 more views at Waseda University in 2015 than Group A (Fig. 6), indicating that Group B can more exhaustively extract ra- tionales because the CAM extracts rationales based on the relationships of stakeholders. In the same way as RQ1, we perform Mann-Whitney's U test (Wilcoxon rank sum test). Table 2 shows the results of Mann-Whitney's U test of the number of views. The differences are not statistically significant at the $\mathrm{p}=0.05$ level in Table 2 . However, regarding the average, the GQM+Strategies and CAM teams (i.e. Group B) have higher averages than the GQM+Strategies teams (i.e. Group A) in Fig. 8. According to these results, it seems that GQM+Strategies with CAM could extract rationales more exhaustively than GQM+Strategies alone.

Even though the experimental time at Waseda University in 2014 was very limited, Group B resulted in more views than Group A. Therefore, new rationales can be extracted exhaustively using the CAM regardless of time.

\section{RQ3: Does using GQM+Strategies with CAM ex- tract higher quality rationales than using only GQM+Strategies?}

In addition to extracting fewer rationales than Group $A$, the quality of the extracted rationales was $10 \%$ less for Group B at Waseda University in 2014. However, Group B resulted in a $4 \%$ higher quality at Shimane University and $31 \%$ higher quality at Waseda University in 2015 than Group A (Table 3). These results show that GQM+Strategies with CAM can extract rationales of the same quality as GQM+Strategies alone, but the extraction can be affected by time and the team's ability. 


\section{Case Study 2: Application of GQM+Strategies and CAM in Housing Related Company}

\subsection{Evaluation Design}

To answer RQ4, we introduced GQM+Strategies as well as CAM to the Recruit Sumai Company Ltd. Our three members held three workshops in which four members of the new business development department (Recruit members) attended. During the first workshop, which lasted two hours, we taught the participants how to apply GQM+Strategies to organizations. At the second two-hour workshop, the Recruit members applied GQM+Strategies to their company in order to characterize their business environment, set business goals, and develop strategies. They created a GQM+Strategies grid containing goals, strategies, and rationales on a whiteboard. They also considered the rationales from the stakeholders' viewpoints. After the second workshop, we mapped their rationales extracted into CAM. Then we extracted new rationales and new strategies, as well as considered the rationales from the stakeholders' viewpoints. At the third workshop, we shared the new rationales and strategies with the Recruit members. They checked and accepted the missing rationales.

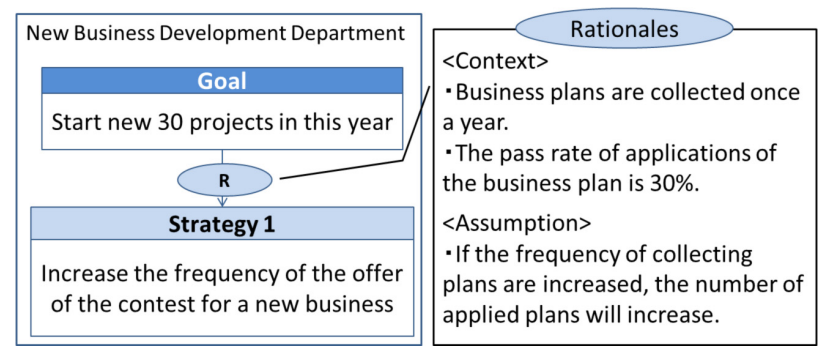

Fig. 9 Part of the GQM+Strategies Grid of the Recruit Sumai Company before using CAM (The numbers in the goals, strategies and rationales differ from the actual ones.)

\subsection{Experimental Result}

At the second workshop, the Recruit members applied GQM+Strategies to their company in order to characterize their business environment, set business goals, and develop strategies. The deliverable was a GQM+Strategies grid (goals and strategies, rationales) on a whiteboard.As a result, they extracted eight rationales, seven goals, and sixteen strategies. They also considered the rationales from the four stakeholders' viewpoints.

After the second workshop, we mapped their rationales using the CAM to verify if rationales were omitted. Then we extracted new ten rationales and new three strategies, as well as considered the rationales from the stakeholders' viewpoints.

Figure 10 shows part of the CAM of the Recruit Sumai Company where black denotes rationales that they extracted (i.e., rationales considered from the business development department's viewpoint). Only Strategy 1 was extracted based on these rationales in Fig. 9. They believed that if they "increase the frequency of the offer of the contest for a new business", they would achieve the business goal of "start 30 new projects this year". However, italics in Fig. 10 denotes new rationales due to the CAM, which were considered from another viewpoint (i.e., applicants' point of view).

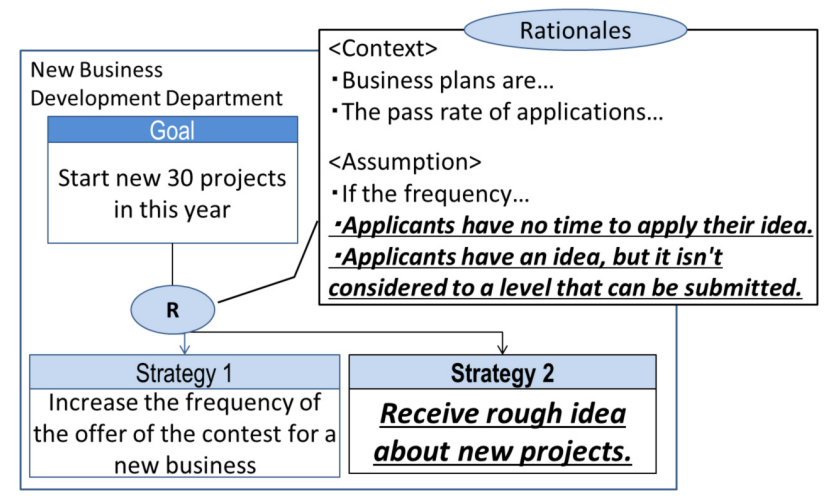

Fig. 11 Part of the GQM+Strategies Grid of the Recruit Sumai Company after using CAM (The numbers in the goals, strategies and rationales differ from the actual ones.)

\begin{tabular}{|c|l|l|l|}
\hline Vietor & \multicolumn{1}{|c|}{ Applicants } & $\begin{array}{c}\text { Judge } \\
\text { (New business } \\
\text { develop Dpt.) }\end{array}$ & \multicolumn{1}{c|}{$\begin{array}{c}\text { Adviser } \\
\text { (New business develop Dpt.) }\end{array}$} \\
\hline Applicants & $\begin{array}{l}\text { A1:Applicants have no time to apply their idea. } \\
\text { A2:Applicants have an idea, but it isn't } \\
\text { considered to a level that can be submitted. }\end{array}$ & & $\begin{array}{l}\text { A3:If same person provide feedback } \\
\text { to participant before and after the } \\
\text { contest, a proposal will be better. }\end{array}$ \\
\hline $\begin{array}{c}\text { Judge } \\
\text { (New business } \\
\text { develop Dpt.) }\end{array}$ & $\begin{array}{l}\text { C1: } 40 \% \text { of the applications can pass. } \\
\text { A4:If frequency of collecting ideas are } \\
\text { increased, number of applications will increase }\end{array}$ & $\begin{array}{l}\text { A5:If rejected ideas are provided } \\
\text { feedback and are refined, they can } \\
\text { pass the context. }\end{array}$ \\
\hline $\begin{array}{c}\text { Adviser } \\
\text { (New business } \\
\text { develop Dpt.) }\end{array}$ & & $\begin{array}{l}\text { A6: An adviser have no time to } \\
\text { advise applicants. }\end{array}$ \\
\hline
\end{tabular}

Fig. 10 Part of the CAM of the Recruit Sumai Company (The numbers in the rationales differ from the actual ones.) 
In fact, the applicants do not have time to revise their ideas if their idea was not considered to be sufficient during a previous contest. If the company implements Strategy 1 , its business goal will not be achieved. Based on the new rationales, we extracted new Strategy 2, "receive rough idea about new projects" (Fig. 11). At the third workshop, we shared the new rationales and strategies with the Recruit members. They checked and accepted the missing rationales. They also noticed that there was a lack of rationales using just a GQM+Strategies grid.

\subsection{Discussion}

\section{RQ4: Can using GQM+Strategies with CAM support to planning the actual goals and strategies in an organization?}

Rationales extracted ad hoc tend to come from limited viewpoints, preventing all rationales from being determined, which may result in deriving incorrect strategies. Thus, rationales must be considered from all the stakeholders' viewpoints. The CAM can be used to consider many different points of view.

In the future, we plan to review the results of the implementation in order to evaluate rationales and strategies. Moreover, we plan to confirm that the CAM can respond to changes in the management policy or business environment.

\section{Limitations}

One threat to the internal validity is the difference between the students' ability by team. To remove this, we conducted three experiments where each experiment involved a different group of students at two universities. The same results were obtained when sufficient experimental time was allotted. Another threat to the internal validity is the difference between the subjects' experiences. However, all students in this study learned GQM+Strategies and the CAM for the first time, and had limited business knowledge. Therefore, this bias was removed.

We conducted three experiments involving students enrolled in a software engineering class with limited business knowledge. Our approach may not have much effect on business professionals with experience. This is a threat to the external validity. To confirm the effectiveness of CAM on business, we introduced GQM+Strategies with CAM to an actual company related to housing. The CAM helped consider different points of view, but the experiment was limited to one domain (a housing related company). Because the CAM has a hierarchy corresponding to the corporate structure, it is possible that the CAM also corresponds to other domains. In the future, we plan to verify the effectiveness of the CAM for other organizations.

\section{Related Work}

The GQM+Strategies approach extends the goal /question /metric paradigm [6], [7], which is a goal oriented approach. Previous research has proposed various approaches to execute a goal-oriented approach.

E. Yu has proposed the $i^{*}$ framework [18], [19], which describes the dependent relationships among various actors in an organizational context. These relationships are used to describe stakeholder interests and concerns, and how they might be addressed by various configurations of systems and environments [20]. Moreover, an actor relationship matrix analysis method (ARM) extends the $i$ *framework. ARM helps ensure the completeness of the requirements in a repeatable and systematic manner that does not currently exist in the $i^{*}$ framework [21]. In this study, we use the point of analyzing requirements from the relationships between stakeholders as a reference for our approach.

Another approach that combines GQM+Strategies and other methods is Utilizing GQM+Strategies for Business Value Analysis [15]. This method integrates GQM+Strategies and Business Value Analysis by coupling cost-benefit and risk analysis (value goals) with operationally measurable business goals, helping to evaluate the success of the business goal and the effectiveness of the chosen strategies. However, whether this method efficiently and exhaustively extracts rationales is unclear. V. Basili et al. have applied the GQM+Strategies approach to ECOPETROL, a global player in the oil and gas industry, for measurement-based ITbusiness alignment [9]. ECOPETROL has been extended to collect and analyze data based upon questionnaires. Moreover, J. Münch et al. have applied the GQM+Strategies method to examine and align the strategic, tactical, and operational goals in software-intensive integrated product development [22].

Our approach has been applied to an example company, but we did not consider operation and maintenance. In the future, we intend to verify whether GQM+Strategies models using CAM can be used for refinement and maintenance.

\section{Conclusion and Future Work}

Typically the biggest factor for a project's failure is insufficient requirements [23]. In our GQM+Strategies with CAM approach, rationales are extracted efficiently and exhaustively by analyzing the relationships of stakeholders in an organization. Experiments involving 43 students at Shimane University and $28+32$ students at Waseda University in Japan demonstrate that GQM+Strategies with CAM can extract rationales more efficiently and exhaustively than using only GQM+Strategies.

In the future, we plan to apply the CAM to other examples in order to validate its flexibility. We will also conduct experiments to derive contexts, assumptions, and strategies for the goals at higher levels of the organizational structure or for multiple levels simultaneously. To verify that the CAM, extracted rationales, and extracted strategies respond to changes in the management policy and business environment, we intend to analyze the implementation results of the Recruit Sumai Company. 


\section{Acknowledgments}

We are grateful to members of the Goal-oriented Quantitative Management Research Group (GQM-RG), who provided insightful comments and suggestions. In addition, we are thankful for Dr. Jens Heidrich's feedback and valuable comments. We are also grateful to the Recruit Sumai Company for their participation in our case study.

\section{References}

[1] T. Kobori, H. Washizaki, Y. Fukazawa, D. Hirabayashi, K. Shintani, Y. Okazaki, and Y. Kikushima, "Efficient identification of rationales by stakeholder relationship analysis to refine and maintain gqm+strategies models," in Proceedings of the 1st Asia Pacific Requirements Engineering Symposium (APRES2014), Communications in Computer and Information Science, vol.432, pp.77-82, Springer Berlin Heidelberg, 2014.

[2] T. Kobori, H. Washizaki, Y. Fukazawa, D. Hirabayashi, K. Shintani, Y. Okazaki, and Y. Kikushima, "Identifying rationales of strategies by stakeholder relationship analysis to refine and maintain gqm+strategies models," in Proceedings of the 15th International Conference of Product Focused Software Development and Process Improvement, Lecture Notes in Computer Science, vol.8892, pp.78-92, Springer International Publishing, 2014.

[3] A. Trendowicz, J. Heidrich, and K. Shintani, "Aligning software projects with business objectives," Software Measurement, 2011 Joint Conference of the 21st Int'1 Workshop on and 6th Int'1 Conference on Software Process and Product Measurement (IWSM-MENSURA), pp.142-150, Nov. 2011.

[4] V. Mandić, L. Harjumaa, J. Markkula, and M. Oivo, "Early empirical assessment of the practical value of gqm+ strategies," Proceedings of the 2010 International Conference on New Modeling Concepts for Today's Software Processes: Software Process, ICSP'10, vol.6195, pp.14-25, Springer-Verlag, 2010.

[5] V. Basili, J. Heidrich, M. Lindvall, J. Münch, M. Regardie, D. Rombach, C. Seaman, and A. Trendowicz, "Bridging the gap between business strategy and software development," ICIS 2007 Proceedings, p.25, 2007

[6] V.R. Basili, M. Lindvall, M. Regardie, C. Seaman, J. Heidrich, J. Munch, D. Rombach, and A. Trendowicz, "Linking software development and business strategy through measurement," Computer, vol.43, no.4, pp.57-65, April 2010.

[7] T. Kaneko, M. Katahira, Y. Miyamoto, and M. Kowalczyk, "Appli-

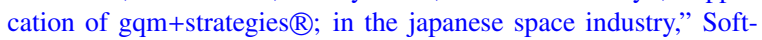
ware Measurement, 2011 Joint Conference of the 21st Int'1 Workshop on and 6th Int'l Conference on Software Process and Product Measurement (IWSM-MENSURA), pp.221-226, Nov. 2011.

[8] V. Basili, C. Lampasona, and A.E.O. Ramírez, "Aligning corporate and it goals and strategies in the oil and gas industry," in ProductFocused Software Process Improvement, Lecture Notes in Computer Science, vol.7983, pp.184-198, Springer Berlin Heidelberg, 2013.

[9] S.A. Sarcia, "Is gqm+strategies really applicable as is to non-software development domains?," Proceedings of the 2010 ACM-IEEE International Symposium on Empirical Software Engineering and Measurement, ESEM '10, pp.45:1-45:4, 2010.

[10] R.S. Kaplan and D.P. Norton, "The balanced scorecard: measures that drive performance," Harvard business review, vol.83, no.7, p.172, 2005.

[11] "Gqm+strategies- the fraunhofer cese." Web site: http://www.fc-md.umd.edu/. Retrieved Nov. 10, 2015.

[12] "Gqm+strategies- fraunhofer iese- fraunhofer-gesellschaft." Web site: http://www.iese.fraunhofer.de/de/produkte/gqm.html. Retrieved Nov. 10, 2015.
[13] R. van Solingen, V. Basili, G. Caldiera, and H.D. Rombach, "Goal question metric (gqm) approach," Encyclopedia of Software Engineering, 2002.

[14] T. Hall and N. Fenton, "Implementing effective software metrics programs," Software, IEEE, vol.14, no.2, pp.55-65, March 1997.

[15] V. Mandić, V. Basili, L. Harjumaa, M. Oivo, and J. Markkula, "Utilizing gqm+strategies for business value analysis: An approach for evaluating business goals," Proceedings of the 2010 ACM-IEEE International Symposium on Empirical Software Engineering and Measurement, ESEM '10, pp.20:1-20:10, ACM, 2010.

[16] V. Basili, A. Trendowicz, M. Kowalczyk, J. Heidrich, C. Seaman, J. Münch, and D. Rombach, Aligning Organizations Through Measurement: The GQM+ Strategies Approach, pp.9-17, Springer, 2014.

[17] V. Mandić and M. Oivo, "Sas: A tool for the gqm+strategies grid derivation process," in Product-Focused Software Process Improvement, Lecture Notes in Computer Science, vol.6156, pp.291-305, Springer Berlin Heidelberg, 2010.

[18] "i* intentional strategic actor relationships modelling? istar." Web site: http://www.cs.toronto.edu/km/istar/. Retrieved Nov. 10, 2015.

[19] E. Yu, "Conceptual modeling: Foundations and applications," ch. Social Modeling and I*, pp.99-121, Springer Berlin Heidelberg, 2009.

[20] E.S.K. Yu, "Towards modelling and reasoning support for early-phase requirements engineering," Proceedings of the Third IEEE International Symposium on Requirements Engineering, 1997, pp.226-235, Jan. 1997.

[21] S. Yamamoto, K. Ibe, J. Verner, K. Cox, and S. Bleistein, "Actor relationship analysis for the $\mathrm{i}^{*}$ framework," in Enterprise Information Systems, Lecture Notes in Business Information Processing, vol.24, pp.491-500, Springer Berlin Heidelberg, 2009.

[22] J. Munch, F. Fagerholm, P. Kettunen, M. Pagels, and J. Partanen, "Experiences and insights from applying gqm+strategies in a systems product development organisation," 2013 39th EUROMICRO Conference on Software Engineering and Advanced Applications (SEAA), pp.70-77, Sept. 2013.

[23] C. Ebert, "Requirements before the requirements: understanding the upstream impacts," Proceedings 13th IEEE International Conference on Requirements Engineering, 2005, pp.117-124, Aug. 2005.

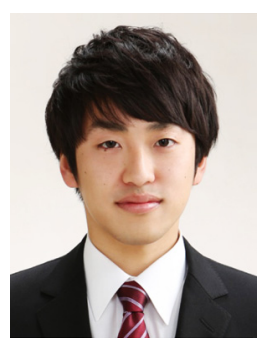

Takanobu Kobori received the B.E. degree in Information and Computer Science from Waseda University, Tokyo, Japan in 2014. He is now a master course student of Department of Information and Computer Science, Waseda University. His research interests include software engineering especially requirement engineering. 


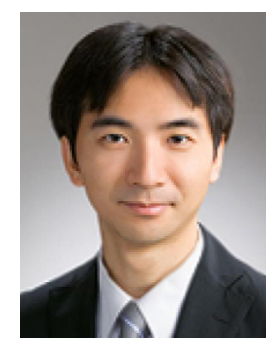

Hironori Washizaki is a professor at Waseda University, Tokyo, Japan. He is also a visiting professor at National Institute of Informatics, Tokyo, Japan. He obtained his Doctor's degree in Information and Computer Science from Waseda University in 2003. His research interests include software reuse, patterns and quality assurance. He has served as members of program committee for many international conferences including ASE, SEKE, PROFES, APSEC and PLoP. He has also served as members of editorial board for several journals including Journal of Information Processing. He is serving as outside director at SYSTEM INFORMATION CO., LTD. since December 2015.

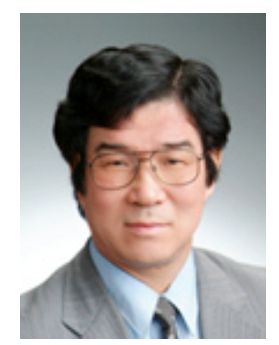

Yoshiaki Fukazawa received the B.E., M.E. and D.E. degrees in electrical engineering from Waseda University, Tokyo, Japan in 1976, 1978 and 1986, respectively. He is now a professor of Department of Information and Computer Science, Waseda University. Also he is Director, Institute of Open Source Software, Waseda University. His research interests include software engineering especially reuse of object oriented software and agent-based software.

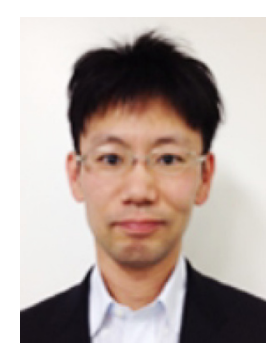

Daisuke Hirabayashi

is a researcher at Goal-oriented quantitative management research group, Waseda University, Japan. He is working for T\&D INFORMATION SYSTEM Ltd.

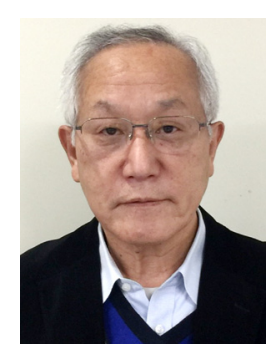

Katsutoshi Shintani

is a researcher at Goal-oriented quantitative management research group, Waseda University, Japan. He is running Shintani IT consulting.

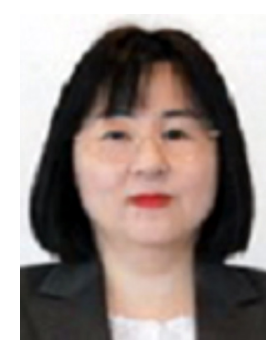

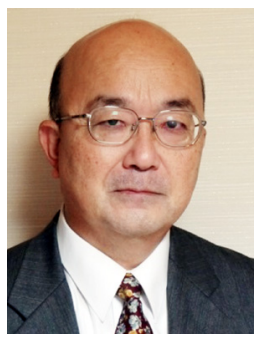

Yasuhiro Kikushima is a researcher at Goal-oriented quantitative management research group, Waseda University, Japan. 